\title{
LASER-VISUAL-INERTIAL ODOMETRY BASED SOLUTION FOR 3D HERITAGE MODELING: THE SANCTUARY OF THE BLESSED VIRGIN OF TROMPONE
}

\author{
G. P. C. Bronzino ${ }^{1}$, N. Grasso ${ }^{2, *}$, F. Matrone ${ }^{2}$, A. Osello ${ }^{3}$, M. Piras ${ }^{2}$ \\ ${ }^{1}$ Scuola di Specializzazione in Beni Architettonici e del Paesaggio - Politecnico di Torino Corso Duca degli Abruzzi 24, 10129 \\ Torino (Italy) (giosuepiercarlo.bronzino@studenti.polito.it) \\ ${ }^{3}$ Department of Environment Land and Infrastructure Engineering (DIATI) - Politecnico di Torino Corso Duca degli Abruzzi 24, \\ 10129 Torino (Italy) (nives.grasso@polito.it; francesca.matrone@polito.it; marco.piras@polito.it) \\ ${ }^{2}$ Department of Structural, Geotechnical and Building Engineerin (DISEG) - Politecnico di Torino Corso Duca degli Abruzzi 24, \\ 10129 Torino (Italy) (anna.osello@polito.it)
}

Commission II, WG II/8

KEY WORDS: LiDAR, Mobile Mapping Systems, SLAM, photogrammetry, restoration, Cultural Heritage

\begin{abstract}
:
The advent of new mobile mapping systems that integrate different sensors has made it easier to acquire multiple 3D information with high speed. Today, technological development has allowed the creation of portable systems particularly suitable for indoor surveys, which mainly integrating LiDAR devices, chambers and inertial platforms, make it possible to create in a fast and easy way, full 3D model of the environment. However, the performance of these instruments differs depending on the acquisition context (indoor and outdoor), the characteristics of the scene (for example lighting, the presence of objects and people, reflecting surfaces, textures) and, above all, the mapping and localization algorithms implemented in devices. The purpose of this study is to analyse the results, and their accuracy, deriving from a survey conducted with the KAARTA Stencil 2 handheld system. This instrument, composed of a 3D LiDAR Velodyne VLP-16, a MEMS inertial platform and a feature tracker camera, it is able to realize the temporal 3D map of the environment. Specifically, the acquisition tests were carried out in a context of metrical documentation of an architectural heritage, in order extract architectural detail for the future reconstruction of virtual and augmented reality environments and for Historical Building Information Modeling purposes. The achieved results were analysed and the discrepancies from some reference LiDAR data are computed for a final evaluation. The system was tested in the church and cloister of the Sanctuary of the Beata Vergine del Trompone in Moncrivello (VC) (Italy).
\end{abstract}

\section{INTRODUCTION}

In the last decade, advanced Geomatics researches revealed the advantages of using integrated solutions and multi-sensor strategies for the digital documentation of complex environments obtaining improved 3D models of articulated structures in the heritage domain. Multi-sensor image-based and range-based techniques, as close-range photogrammetry (CRP) and terrestrial laser scanning (TLS), are consolidated approaches for the architectural multi-scale and multi-resolution reconstruction and analysis. These 3D models do not only support metric documentation, analyses on the state of conservation, structural recovery and architectural restoration, but are the basis of innovative solutions of virtual reality (VR), augmented reality (AR), holograms and video mapping to develop and test new communication opportunities suitable for modern forms of walking and visiting places, in addition to BIM (Khoshelham et al., 2017). To these last purposes, it is demanded to the Geomatics to provide easy-to-use and easy-manageable highresolution models made of dense point clouds and textured meshes.

In the geomatics field discussions are still opened on which is the more suitable technique to use for 3D modeling purposes, but one of the answers to this issue could be the combination of different strategies and the integration of data deriving from different sensors (Remondino et al., 2010) especially when surveying a complex site. As it is well known, consolidated surveying approaches often require time spending operations, concerning especially the planning of the surveys, manoeuvrability matters and the data acquisition phase. Moreover, the so realized point clouds are extremely dense and require further processing in order to be filtered from noise, subsampled and simplified for 3D modelling or visualization purposes. In particular, when the context of analysis is the indoor environment, as in this study, the effectiveness of 3D data image or range measurement systems should be assessed according to the context and based on the descriptive capabilities obtainable in environments consisting of complex geometries and particular materials to obtain an adequate reconstruction of the surface in response to the proposed aims.

In order to overcome these issues, several handheld solutions for rapid and close-range mapping systems are now available on the market, with the name "indoor mobile mapping systems" (IMMSs) (Keller and Sternberg, 2013), which combine multiple sensors, both active, passive and inertial, in a unique and easyportable platform. This technology is flexible enough to be used in several application fields, but is particularly suitable for indoor mapping of buildings (Nocerino et al., 2017; Lagüela et al., 2018). The IMMS technological solutions differ from each other in the types and performances of the integrated sensors, in the acquisition range, but also in the 3D mapping algorithms that are implemented. Generally, these tools aim to overcome the problem of localization through the analytical method of "simultaneous localization and mapping (SLAM) (Dissanayake et al., 2001; Nüchter et al., 2007), which simultaneously allows to estimate the position of the instrument and generate a digital model of the scene detected (Tucci et al., 2018). The IMMS available on the market are now countless and several research studies aim to evaluate their behavior and performances. (Thomson et al., 2013, Lehtola et al., 2017, Chiabrando et al.,

\footnotetext{
* Corresponding author
} 
2018; Sammartano and Spanò, 2018, Tucci et al., 2018). The presented research faced with the verification of the accuracy and richness of the information content of the point clouds generated by the KAARTA Stencil 2 in the stand-alone solution, that allows to obtain 3D models in real time, without the need to reprocess the data. The main advantage of this tool is the use of a new visual-inertial odometry algorithm (Zhang and Singh, 2018). In this contribution, the method for evaluating the point clouds obtained with the handheld system and the resulting differences with a reference data was described.

\subsection{The case study}

The case study examined is the Sanctuary of the Beata Vergine del Trompone in Moncrivello (VC) and its restoration process has become a precious opportunity for the training and the experimentation of several lines of research, from HBIM models to VR and AR, therefore the surveying activity of this complex is aimed at different purposes.

In particular, in anticipation of an imminent restoration intervention, aimed at the preservation of the existing building, a systematic and accurate survey campaign was carried out and the construction of an HBIM of the whole complex is in progress. In this framework, the Stencil Kaarta point clouds could be really useful, once coloured, to speed-up the reconstruction process of $\mathrm{VR}$ and AR environments, as for these purposes it is not requested a very high level of detail and accuracy. This was one of the main reasons to test this instrument, in addition to the possibility to survey an extensive architectural heritage quickly and with the integration of less instruments as possible.

The survey process, as well as the entire study, is part of the collaboration between the Politecnico di Torino, with the Drawing to the future laboratory of the DISEG department and the surveying group of the DIATI department, the Cultural Heritage Office of the Arcidiocesi of Vercelli. The Sanctuary has been entrusted, since 1970, to an international association of faithful people, which, in addition to health care, take care of the opening of the church. The partnership between the three entities mentioned above derives from the desire to restore the religious buildings that make up the Sanctuary (church, Rotonda, presbytery, sacristy and bell tower) which unfortunately suffers the effects of a protracted lack of maintenance on building envelopes and windows. The intervention aims not only at the restoration of buildings but also to realize a process of valorization of the Sanctuary with virtual and augmented reality systems (VR and AR).

The Sanctuary was built in 1562 and, over the centuries, a set of buildings has been added with multiple functions (Figure 1), sometimes even monastic ones, today switched to health care and assistance (Archivio della Curia Arcivescovile di Milano, 1584; Pauna, 1895; Bono, 1935; Pissinis and Carando, 1988; Cappellino, 1995).

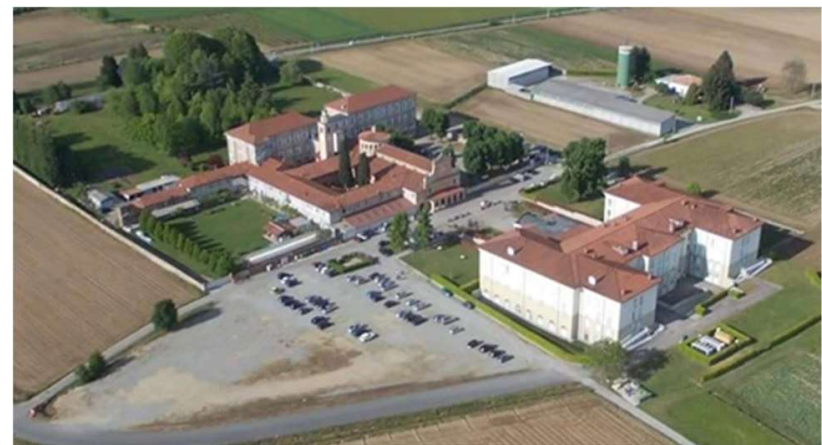

Figure 1 . The whole complex with the health care building on the right and the Church and cloister on the left.
The church (Figure 2a) is composed of building parts dating back to different eras but in harmony with each other. The oldest portion is the so-called "Rotonda" (rounded), the central plan building ended in 1568 which rises higher than the other bodies. On the main axis of the first building a rectangular hall was built, divided into three naves by two rows of stone columns. The central nave, which is about $4.5 \mathrm{~m}$ taller than the side aisles, leaves space on both sides for a series of trilobate windows that give light to the interior (Figure $2 b$ ).

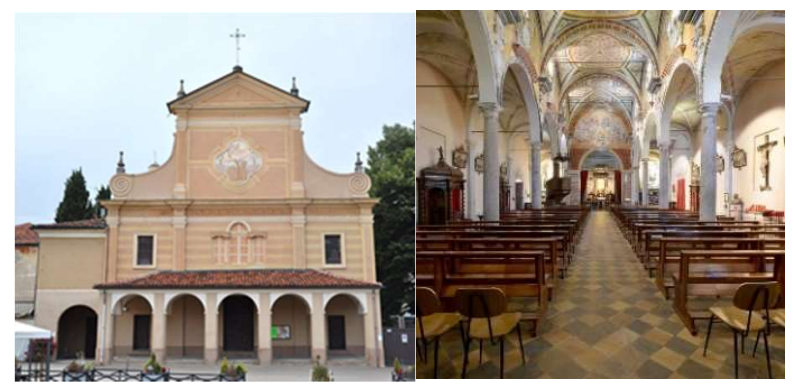

Figure $2 \mathrm{a}$ and $\mathrm{b}$. Facade and naves of the Church.

Accessing the Sanctuary by means of the central entrance, two rows of stone columns in the Tuscan style divide the hall into three naves. The aisles are surmounted by cross vaults and are crossed by pilasters leaning against the side walls of the building. All the vaults have been the object of pictorial decoration, more refined in the central nave.

\section{THE ACQUISITION METHODOLOGY}

\subsection{The Kaarta Stencil 2}

The 3D rapid mapping standalone system, KAARTA Stencil 2 (Figure 3), is a relatively low-cost tool of limited size, able to estimate the position in real time and generate point clouds of the environment with an acquisition range of $100 \mathrm{~m}$.

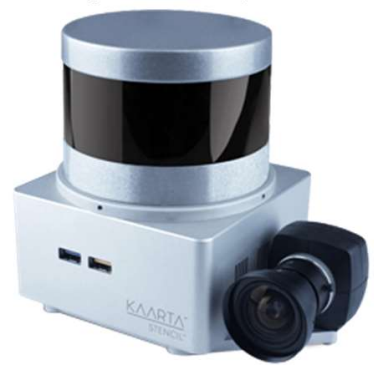

Figure 3. The KAARTA Stencil 2.

The device consists of a 3D LiDAR Velodyne VLP-16, a MEMS inertial platform and a feature tracker camera, connected to an i7 processor. The measurement principle on which the system is based combines laser range data, data from the feature tracker and image information to estimate 6-DOF motion and record the points acquired by the LiDAR between them. Data processing follows a two-step workflow (Zhang and Singh, 2018): first of all, visual-inertial odometry algorithms are exploited, integrating vision and IMU for the optimization of motion estimation, associating, where possible, the information related to the depth of features, using laser points or through triangulation. At the same time, using the speed information deriving from the odometry, the laser points are registered in a local system. A mapping algorithm detects the geometric features in the point cloud and matches them in order to optimize the registration, using the pose constraints deriving from the previous step. This solution therefore allows the use of the instrument using only the 
lidar-odometry, without the need for images for the feature tracking, even if with a greater risk of positioning errors.

This instrument can be integrated with other sensors, such as GNSS receivers and installed on different supports. In this case study, the Stencil 2 was mounted on a small pole and connected to a screen to verify data acquisition in real time.

\subsection{Acquisition tests}

For the analysis of the KAARTA Stencil 2 two acquisition tests were performed, following two different closed paths inside the Sanctuary. The first test field was the inside of the Church, a large indoor environment with furnishing elements, free of moving objects or people and quite well artificially lit. The planned path crossed the three naves, passing through the circular room below the vault, and its end point (E) corresponded to the starting point (S) (Figure 4). The second acquisition has been performed initially along a narrowed corridor inside the sanctuary, which has been travelled twice, in both directions, then, the path, passed through the four sides of the cloister and it was stopped at the same starting point (S) (Figure 4). The second test field showed poor light conditions and several people were moving along the spaces. In both cases the ground was even everywhere.

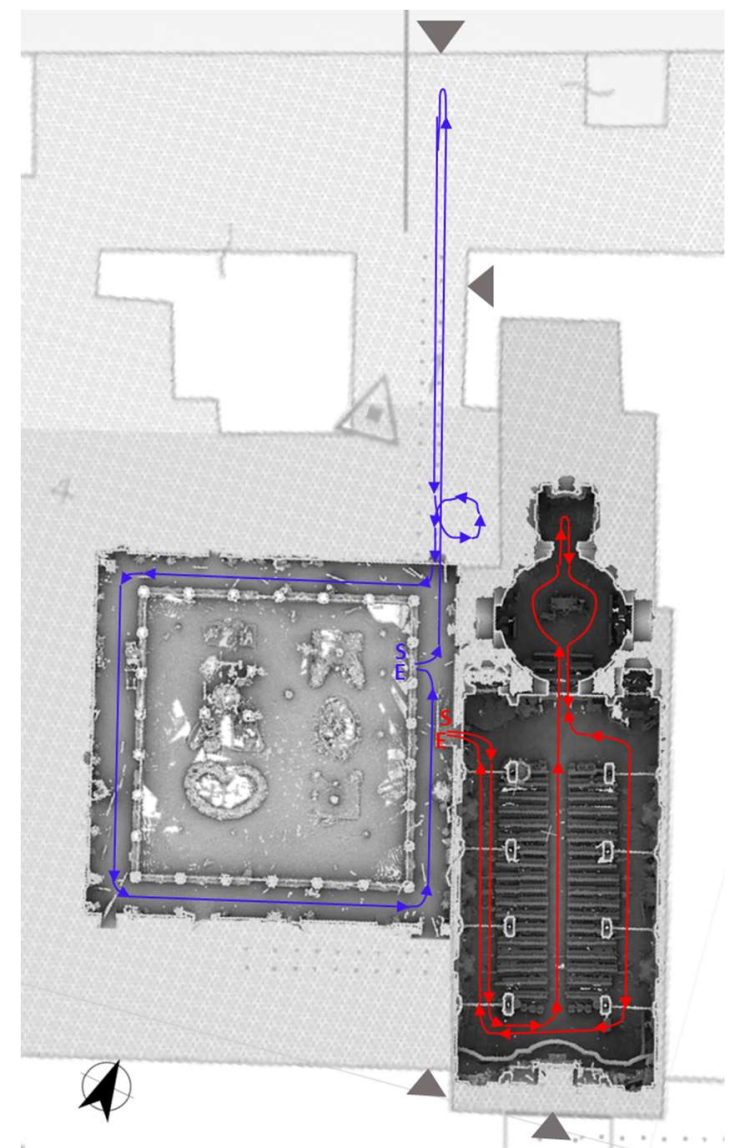

Figure 4. The test paths: in red, the first trajectory followed inside the Curch; in blue, the path defined through the corridor and along the cloister. (S) and (E) represent, respectively, the start and end point.

The data acquisitions were performed using the Stencil 2 default configuration parameters, set in order to use the instrument in structured outdoor environments or large indoor spaces (Kaarta, 2018). Specifically, these settings include default values for the voxel size $(0,2 \mathrm{~m})$, namely the resolution of the point cloud in map file, the resolution of the point cloud for scan matching and display (cornerVoxelSize, surfVoxelSize, sorroundVoxelSize) $(0,2 \mathrm{~m}-0,4 \mathrm{~m}-0,6 \mathrm{~m})$, the minimum distance of the points to be used for the mapping (blindRadius) $(2 \mathrm{~m})$ and no constraints on the planarity of the motion. The Stencil 2 offer the chance of post-process and improve the products by changing the parameters values, thanks to a specific tool; this functionality will be addressed in Section 3.2, in order to assess the effectiveness of the reprocessing tool.

\subsection{The reference data}

In order to predict the accuracy of the 3D data acquired by the Stencil 2, two point clouds, of the Church and the Cloister, created from 3D laser scans, were employed as 3D reference models. A first step to the generation of the ground truth has involved the construction of a topographic network composed of six vertices. The survey was made through a GNSS double frequency and multi-constellation receiver in static modality standing on each point for about 1 hour. Starting from the reference network, it was possible to acquire the position of some reference point using a total station and a prism. All measurements were subsequently adjusted with the MicroSurvey StarNet v.7.0 software, in order to obtain the final coordinates: the root mean square (RMS) of the estimated coordinates is less than $1 \mathrm{~cm}$.

Then, terrestrial laser scanning surveys were performed to obtain a reference model for the spatial information. The georeferencing of the scans took place through the pre-signalization of the 36 markers distributed within the church and cloister, measured by topographic techniques. To describe the entire area, 25 scans were acquired using the laser Faro Cam2 Focus 3D. Each scan was acquired with $1 / 4$ of resolution $(\div 1$ point every $7 \mathrm{~mm}$ at 5 $\mathrm{m}$ ), and it requires about 7 minutes for cloud acquisition and 1 minute for images collection. The laser data were processed using Scene (v. 5.2), which is the own software developed by FARO. The elaboration required about 15 hours for the entire process and the result are two coloured 3D point clouds, one representing the inside of the church and the other related to the cloister, composed of 500 and 330 million points respectively. The Figure 5 shows the final reference models, while the Table 1 reports the characteristics of each reference point cloud.

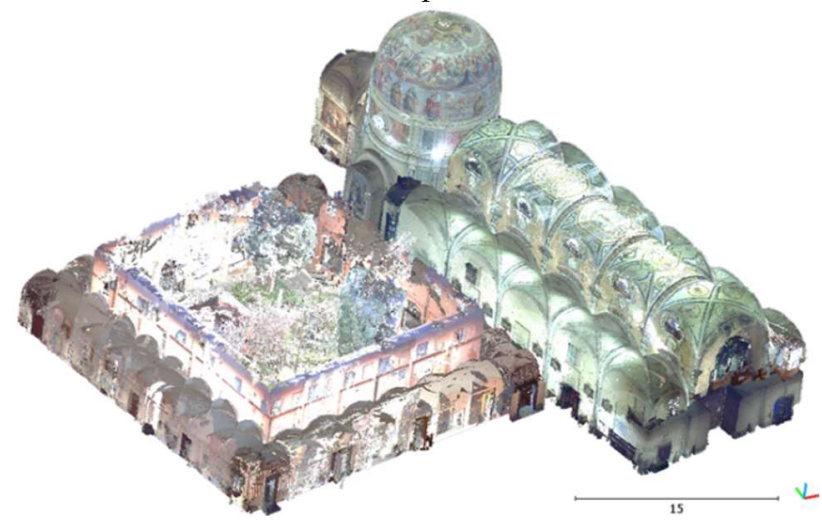

Figure 5. The reference LiDAR point clouds of the Church and the Cloister.

Table 1. Characteristics of the obtained reference point clouds.

\begin{tabular}{ccccc}
\hline $\begin{array}{c}\text { Reference } \\
\text { point cloud }\end{array}$ & $\begin{array}{c}\text { Acquisition } \\
\text { time }\end{array}$ & N. scans & N. points & $\begin{array}{c}\text { Coordinate } \\
\text { System }\end{array}$ \\
\cline { 1 - 4 } Church & 1 day & 12 & $\begin{array}{c}500 \\
\text { million }\end{array}$ & UTM WGS84 \\
\cline { 1 - 4 } Cloister & 1 day & 13 & $\begin{array}{c}330 \\
\text { million }\end{array}$ & $32 \mathrm{~N}$ \\
\hline
\end{tabular}


Due to the high data density of the reference models, point clouds were decimated to clouds with $5 \mathrm{~mm}$ resolution.

\section{DATA ANALYSIS AND POST-PROCESSING}

One of the main advantage in using the KAARTA Stencil 2 is the real-time availability of the data, without the need of further input data, such as GNSS position, reference map of the environment, etc.. At the end of the acquisition phase the point cloud, the estimated trajectory, the acquired images and raw data are stored in the computer. However, the raw data and the images, if used for the localization problem, can be reprocessed, in order to optimize the solution, namely the 3D map generation and the trajectory estimation. In this section, we aimed at assessing the completeness and accuracy of the data deriving both from the real-time and post-elaborated solution, by comparing them to the reference model. Finally, it has been proposed a cloud colouring solution of the Stencil 2 point cloud, using some images acquire by a spherical camera.

\subsection{The real time solution}

Table 2 resumes the main characteristics of the resulting products of the Stencil 2 acquisitions, while in Figure 6, Figure 7 and Figure 8 are showed the captured data, both the point clouds and the trajectories.

Table 2. Characteristics of the real-time solutions of the KAARTA Stencil 2.

\begin{tabular}{|c|c|c|c|c|c|}
\hline \multirow[b]{2}{*}{ Data } & \multirow[b]{2}{*}{$\begin{array}{c}\text { Acquisition } \\
\text { time [s] }\end{array}$} & \multicolumn{2}{|c|}{ Trajectory } & \multicolumn{2}{|c|}{ 3D Point cloud } \\
\hline & & $\begin{array}{c}\text { N. } \\
\text { Points }\end{array}$ & $\begin{array}{l}\text { Length } \\
\text { [m] }\end{array}$ & N.Points & $\begin{array}{l}\text { Density } \\
{[\mathbf{p} / \mathbf{m} 2]}\end{array}$ \\
\hline & 22 & 1138 & 159,31 & 16784402 & 12585 \\
\hline Cloister & 244 & 1209 & 204,05 & 14213460 & - \\
\hline
\end{tabular}

The obtained point cloud of the inside of the church (Figure 6) appears to be quite dense, with the exception of the vaults, due to the considerable height of the ceiling and the limited field of view of the instrument.

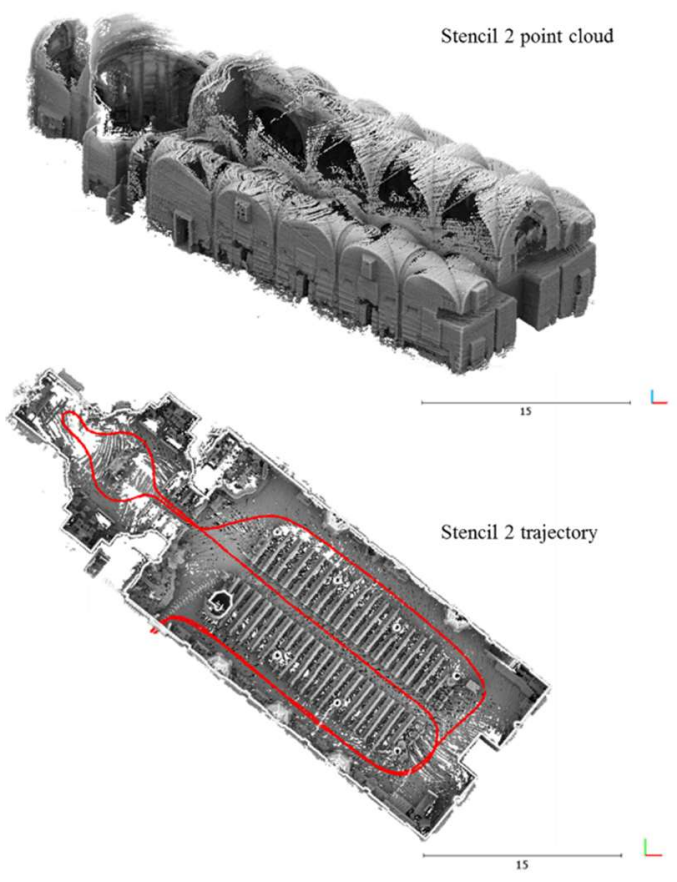

Figure 6. KAARTA Stencil 2 dataset obtained following the first path: the 3D point cloud (top of the image) and the estimated trajectory (lower part of the image).

The observation of this $3 \mathrm{D}$ solution allows to clearly recognize many architectural details, such as columns, doors and windows, and the furniture, like benches, confessionals and the pulpit (Figure 7). From a visual examination of the results deriving from the second acquisition session (Figure 8), it is evident that the point cloud has not been correctly registrated and the trajectory differs considerably from the correct solution, in particular, great errors are noticed in correspondence of the component $\mathrm{z}$ of the motion. These issues were probably caused by the condition of illumination of the corridors, but, most of all, by the presence of people inside the scene, which could affect both the feature matching and the points registration. For these reasons, we considered it useless to estimate the density of the point cloud, since it wouldn't have been a representative datum for the analysis of the instrument performances.

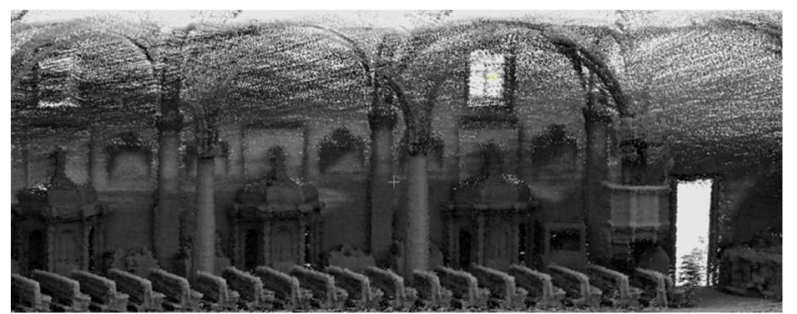

Figure 7. The visible details of the inside of the Church.

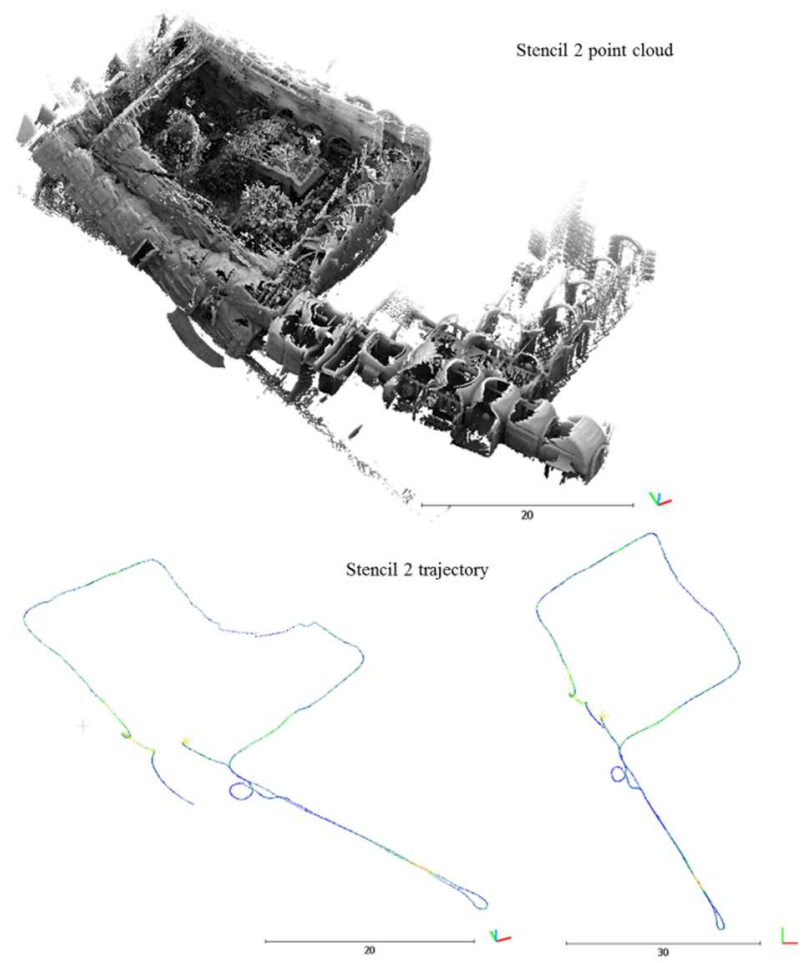

Figure 8. KAARTA Stencil 2 dataset obtained following the second path: the 3D point cloud (top of the image) and the estimated trajectory (lower part of the image).

\subsection{Data post-processing}

As previously anticipated, the last version of the KAARTA Stencil has a dedicated tool for post-processing the acquired data. Specifically, it is possible to reprocess data at a lower speed than 
the original one, in order to improve the registration of point clouds where the instrument may have failed in real-time. In addition, the software allows to modify the configuration parameters and adapt them to the type of environment in which the surveys were carried out.

In order to reprocess the data obtained in the two test fields, firstly, the quality of the images acquired by the feature tracker was improved, increasing the contrast and brightness to facilitate recognition of features.

Therefore, the configuration parameters of the two acquisition tests were modified, as shown in the Table 3 . In both cases, the motion was limited to the x-y plane, since the terrain of the study area was even. Furthermore, the default parameters are set for acquisitions in large indoor environments, therefore, it was considered appropriate to halve the parameters related to the resolution of the point cloud of the second case study, taking into account that it is a tight indoor environment (Kaarta, 2018).

Table 3. Acquisition configuration parameters set for data reprocessing.

\begin{tabular}{lcc}
\hline $\begin{array}{l}\text { Configuration } \\
\text { parameters }\end{array}$ & $\begin{array}{c}\text { 1th path: } \\
\text { Church }\end{array}$ & $\begin{array}{c}\mathbf{2}^{\text {nd }} \text { path: } \\
\text { Cloister }\end{array}$ \\
\hline voxelSize [m] & 0,2 & 0,1 \\
\hline cornerVoxelSize [m] & 0,2 & 0,1 \\
\hline surfVoxelSize [m] & 0,4 & 0,2 \\
\hline sorroundVoxelSize [m] & 0,6 & 0,3 \\
\hline blindRadius [m] & 2 & 1 \\
\hline usePlanarMotion & true & true \\
\hline
\end{tabular}

At the end of the data post-processing, new point cloud and trajectory are automatically stored.

Despite the reprocess, the point cloud acquired following the second path through the cloister still showed evident misalignment and was not taken into consideration for the following analyses. This issue is still probably related to the people moving around the space and the very poor light, which probably produce gross errors in the trajectory estimation and points registration.

\subsection{Comparison of the results}

In order to assess the quality and correspondence to reality of the laser-visual-inertial odometry approach with the Stencil 2, two procedures have been defined: firstly, the distance between the clouds thus generated and the reference cloud was computed. Then, the profile distance, especially in correspondence of corner, has been evaluated.

To this end, the CloudCompare ${ }^{1}$ open source software has been used, which allows these estimates to be performed.

\subsubsection{C2C comparison}

The difference between the reference cloud and the Stencil 2 maps can be estimated using the distance Cloud-to-Cloud (C2C) tool. The $\mathrm{C} 2 \mathrm{C}$ tool exploits the Nearest Neighbour algorithm to compute the Euclidean distance between each point of the compared cloud and the nearest point of the reference cloud.

At this point, it was necessary to scale and register the models generated by Stencil 2 in a reference system consistent with the other model. In this regard, CloudCompare offers several tools: a rough registration can be performed through pairs of equivalent points identified in the point cloud to be registered and in the reference model; subsequently, the automatic method based on the Iterative Closest Point (ICP) algorithm (Besl and McKay, 1992) allows the two models to be very finely recorded. This procedure was performed for both models deriving from the Stencil 2 with a final RMSE of about $20 \mathrm{~mm}$.

The $\mathrm{C} 2 \mathrm{C}$ tool was launched by setting the points distant from the reference model more than $50 \mathrm{~cm}$ as outliers, since the errors that interest us are lower than this limit.

The Figure 9 and Figure 10 shows some significant results deriving from this procedure. The computation of the differences confirmed the metrical accuracy of the models estimated by the Stencil 2, and showed that the greater discrepancies correspond to the floor and vaults surfaces and to the areas where the reference point cloud had no information. Indeed, in correspondence of the floor and the ceiling, the Stencil 2 point cloud is quite sparse and noisy, due to the limited field of view of the instrument.

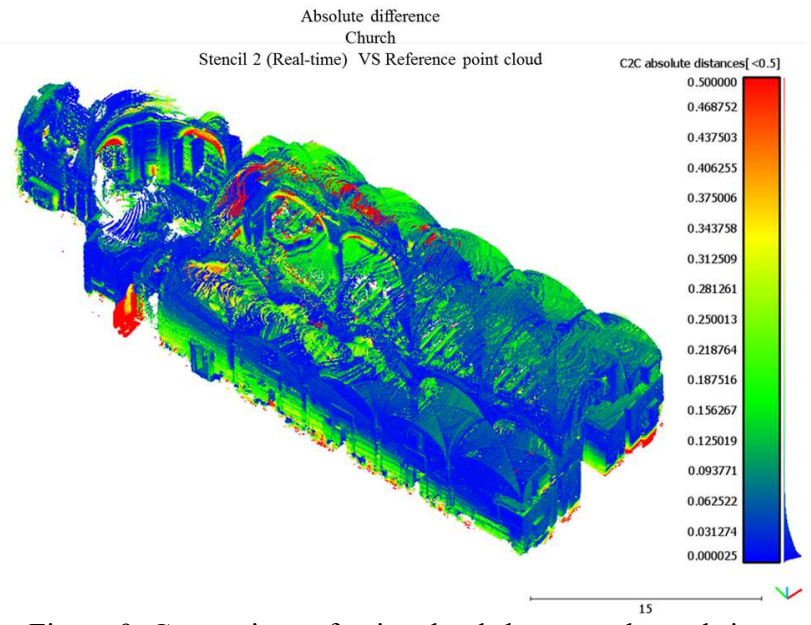

Figure 9. Comparison of point clouds between the real-time solution of the Stencil 2 and the reference model.

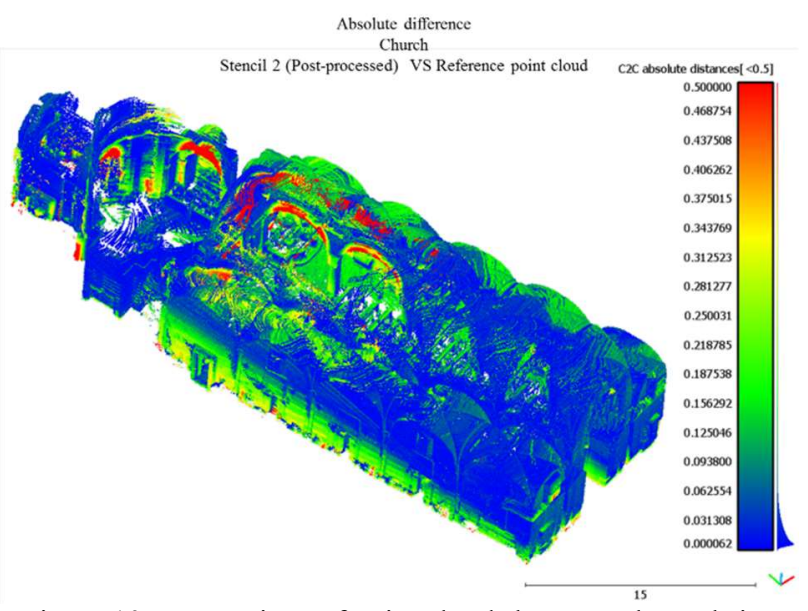

Figure 10. Comparison of point clouds between the real-time solution of the Stencil 2 and the reference model.

In Table 4 are reported the statistical parameters derived from the analysis with $\mathrm{C} 2 \mathrm{C}$ tool, such as maximal distance, average distance and standard deviation.

Table 4. Statistical results from the distance comparison between smartphone point clouds and the reference point cloud.

\begin{tabular}{lccccc}
\hline \multirow{2}{*}{$\begin{array}{c}\text { Distance } \\
\text { computation }\end{array}$} & $\begin{array}{c}\text { Avg. } \\
\text { Distance } \\
{[\mathbf{m}]}\end{array}$ & $\begin{array}{c}\text { Max } \\
\text { distance } \\
{[\mathbf{m}]}\end{array}$ & $\begin{array}{c}\text { RMSE } \\
{[\mathbf{m}]}\end{array}$ & \multicolumn{2}{c}{ Error distribution $[\mathrm{m}]$} \\
\cline { 5 - 6 } Church & 0.045 & 2,740 & 0.074 & $<0.024$ & $<0.060$ \\
\hline
\end{tabular}

\footnotetext{
${ }^{1} \mathrm{http}: / /$ www.cloudcompare.org/
} 


\begin{tabular}{llllll}
\hline Real-time & & & & & \\
$\begin{array}{l}\text { Church } \\
\text { post- } \\
\text { processed }\end{array}$ & 0.032 & 1.863 & 0.059 & $<0.022$ & $<0.056$ \\
\hline
\end{tabular}

From the analysis of these results it emerges the very small difference between the real-time and the post-processed solution. The results obtained show that more of $80 \%$ of the points clouds realized with the Stencil 2 deviate from the reference data less than $6 \mathrm{~cm}$

\subsubsection{Profile comparison}

A second analysis was made by comparing some profile extracted by the Stencil 2 and reference point clouds. Using the specific tool in CloudCompare, two horizontal $5 \mathrm{~cm}$-wide slices and one vertical slice were extracted from each model, respectively at $1,70 \mathrm{~m}$ (eye-level height) and 3,70 $\mathrm{m}$ (Figure 11) height, and in the middle of the central aisle.

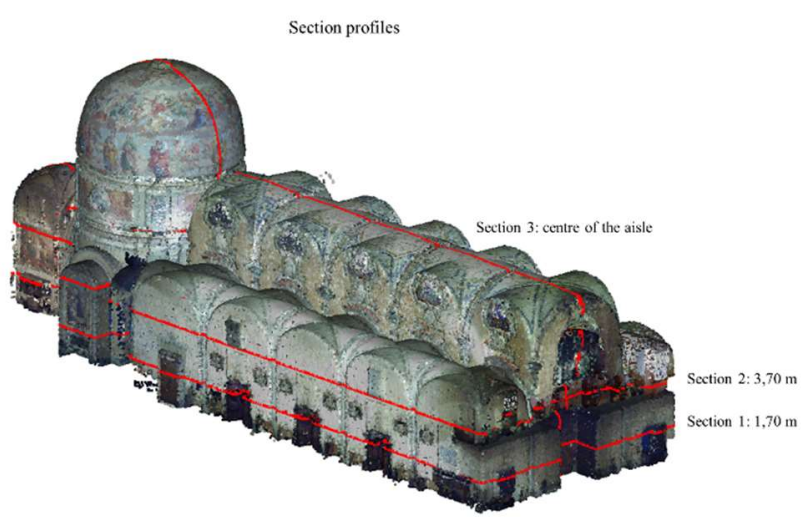

Figure 11. Slices extracted from the reference point cloud.

The evaluation was conducted in order to verify the deviation from the reference point cloud in correspondence of some architectonical elements and its variation as a function of height. The verification of the deviations (Figure 12 and Figure 13) has not only shown the noise that characterizes the clouds acquired with the laser-visual-inertial odometry approach and some misalignments (probably due to an incorrect ICP alignment), but has also highlighted the presence of double surfaces (Figure 12), due to an incorrect registration of the points, which even after a post-processing it was possible to resolve.

\subsection{Colouring of the point cloud}

Despite the density of the point clouds acquired by the Stencil 2 system is sufficient to recognize architectural details of mediumsmall dimensions, such as windows, doors and other architectural details, it was decided to add information content to the 3D clouds by attributing radiometric information to them. The camera integrated in the acquisition system allows you to capture RGB images during the survey; however, this option prevents using the camera for the feature tracking, for which black and white images are required. It has also been noted that the RGB images acquired by Stencil 2 tend to blue in the case of low exposure, while in overexposed areas they appear to be red and therefore require an accurate radiometric calibration.

In order to colour the point cloud, we chose to use some spherical images acquired inside the church with a Ricoh Teta V Ver 1.00 2.1 camera.
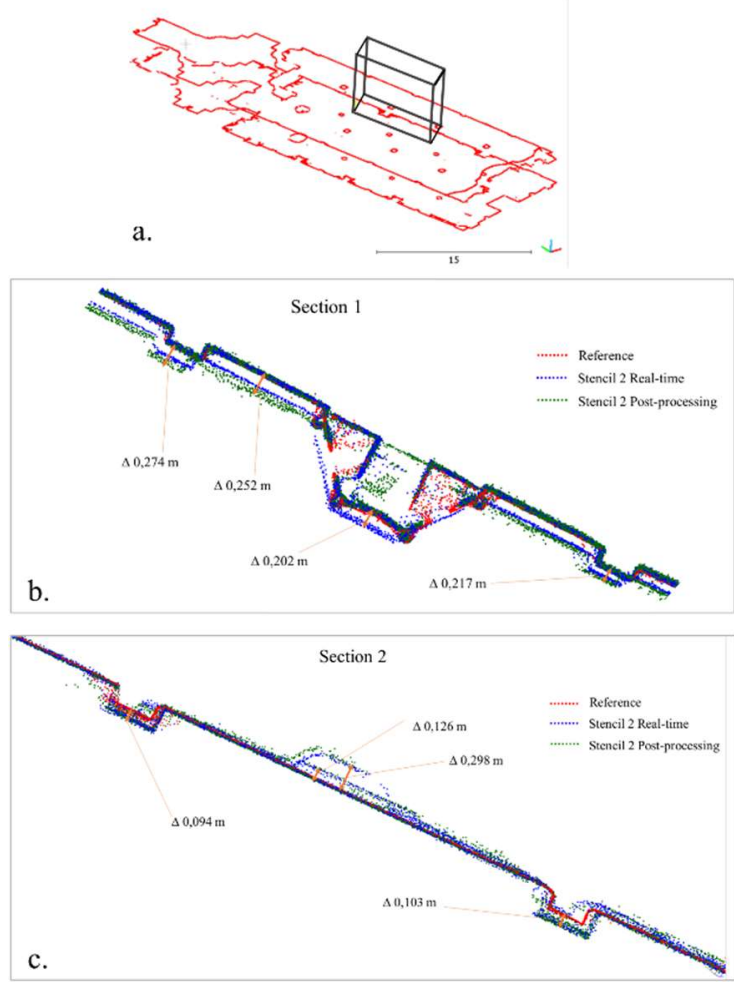

Figure 12. Comparison between the profiles of the reference point cloud, the Real-time Stencil 2 solution and the point cloud obtained after the data reprocessing. In (a.) it are highlighted the

analysed sections; (b.) shows the deviation of the double surfaces at an height of 1,70m; (c.) shows the deviation of the double surfaces at an height of $3,70 \mathrm{~m}$.

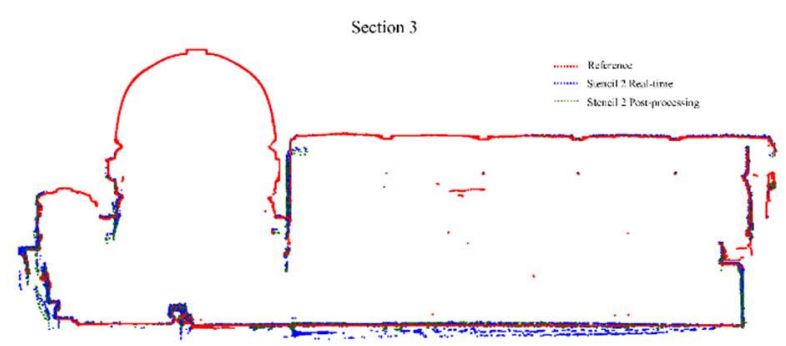

Figure 13. Comparison between the profiles of the reference point cloud, the Real-time Stencil 2 solution and the point cloud obtained after the data reprocessing, along the Section 3.

Through the Agisoft Photoscan Professional software, 78 images were aligned (Figure 14) and then georeferenced using the same markers used for the registration of the clouds acquired with the Faro Focus Cam 3D system. Table 5 shows the characteristics of the resulting dense point cloud and estimated errors on the control points.

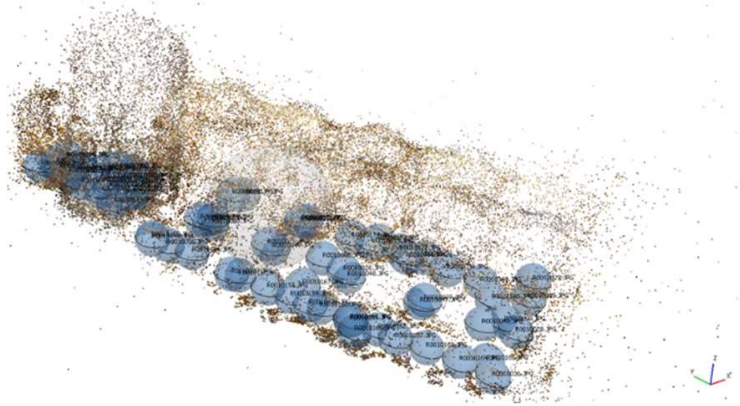

Figure 14. The aligned spherical images. 
Table 5. Characteristics of the generated dense point cloud.

\begin{tabular}{lcccccc}
\hline & & \multicolumn{3}{c}{ Control points } \\
\cline { 5 - 6 } Software & $\begin{array}{c}\text { Aligned } \\
\text { images }\end{array}$ & $\begin{array}{c}\text { Points } \\
\text { dense } \\
\text { point } \\
\text { cloud }\end{array}$ & & $\begin{array}{c}\text { RMSE } \\
(\mathbf{X}) \\
{[\mathbf{m m}]}\end{array}$ & $\begin{array}{c}\text { RMSE } \\
(\mathbf{Y}) \\
{[\mathbf{m m}]}\end{array}$ & $\begin{array}{c}\text { RMSE } \\
(\mathbf{Z}) \\
{[\mathbf{m m}]}\end{array}$ \\
\hline $\begin{array}{l}\text { Agisoft } \\
\text { Metashape } \\
\text { Professional }\end{array}$ & 76 & 13.246 .732 & 0,066 & 0,070 & 0,017 \\
\hline
\end{tabular}

In Figure 15 it is shown the dense point cloud generated from the alignment of the spherical images and characterized by an average ground sample distance of about $8 \mathrm{~mm} /$ pix, considered sufficient taking into account the accuracy of the point cloud acquired with the portable system.

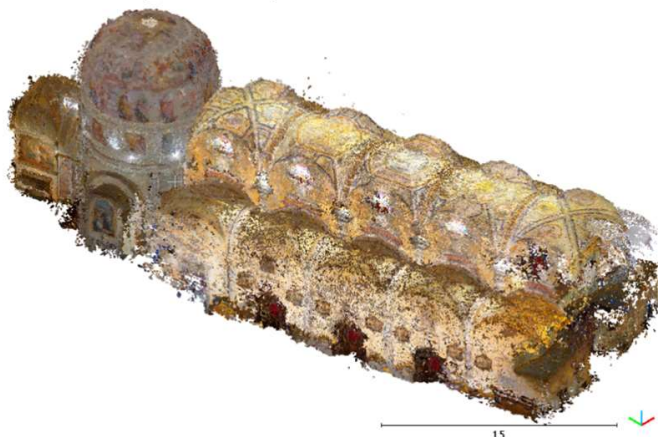

Figure 15. The dense point cloud generated using 76 spherical images.

Using a dedicated tool in CloudCompare it is possible to interpolates the colour of another entity in order to add the radiometric information to the points of a chosen entity. For the test, the point cloud realized after the post-processing of the Stencil 2 data, has been employed. The computation required about 30 seconds to be completed (Figure 16).

Full model
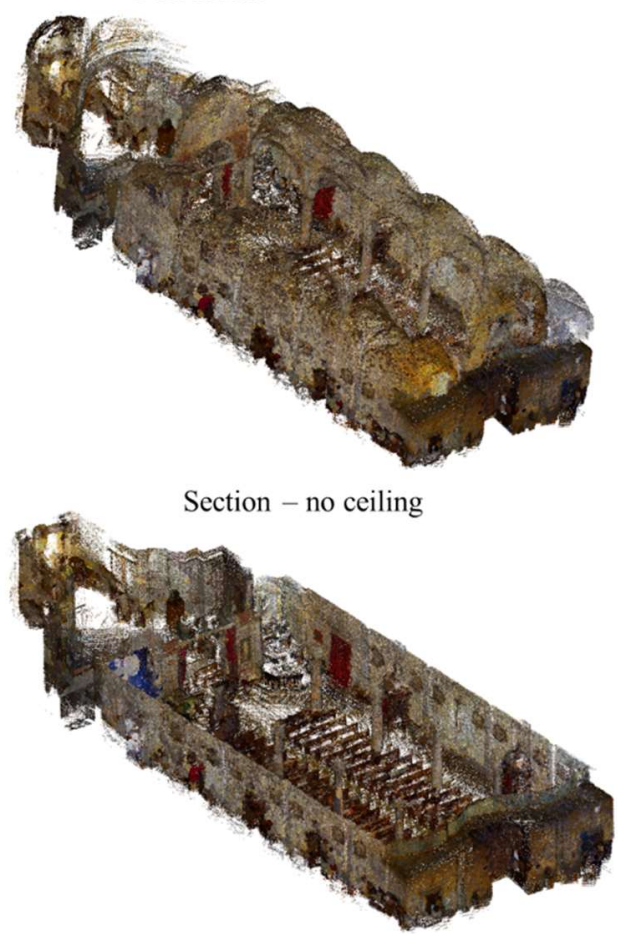

Figure 16. The coloured Stencil 2 post-processed point cloud.

\section{CONCLUSION AND FUTURE WORKS}

The technological development of recent years in the field of geomatics has made portable $3 \mathrm{D}$ rapid mapping tools widely available. These tools differ from each other in the measurement principle which they are based on and the data processing algorithms implemented. In this study the performances of the KAARTA Stencil 2 instrument in a cultural heritage context have been evaluated, which allows to generate 3D maps in real time, without the need for data reprocessing. The tests conducted showed that by combining the information deriving from IMU, laser and feature tracker, it is possible to obtain three-dimensional models with deviations from the reference datum of less than 5 $\mathrm{cm}$. This result could lead at the realization of a high Level of Detail (LOD) in the 3D models as BIM, that could easily overcome the LOD 2 if just a bit higher accuracy is reached, in fact the tolerance of the 1:100 representation scale is $\pm 4 \mathrm{~cm}$. On the contrary, the results can be affected by gross errors if the lighting conditions are limited and above all, in the presence of objects and people moving within the scene. However, it is stressed that this is only a preliminary test; in fact, results cannot be completely generalized, as they are strongly influenced, not only by the characteristics of the environment, but by the setting of the system configuration parameters, as well as by the operator's ability to correctly maneuver the instrument. Analysing qualitatively the obtained products, we can see a good description of the architectural elements and of the objects placed in the scene; however, it has been shown that radiometric information can be added using RGB images, also acquired at different times.

However, it is considered appropriate to carry out further tests, in order to analyse the performances of the instrument following more complex trajectories, in articulated environments, with strong light variations and within structures located on multiple floors.

To date, the problem of georeferencing of the data still remains to be solved, since it is not possible to obtain a detail such as to identify any targets or markers within the scene. Future work will therefore be aimed at testing the effectiveness of the integration of KAARTA Stencil 2 with UltraWideBand (UWB) technology in order to allow direct georeferencing of the point cloud.

Finally, future developments related to the surveying activities of this particular project include:

- the use of the Kaarta point clouds for the construction of a highly detailed BIM in order to experiment innovative technologies in the field of restoration, management and maintenance of a religious complex together with health infrastructures by integrating different disciplines (Figure 17); - experimentation in the field of the most innovative Virtual and Augmented Reality (VAR) technologies for displaying information relating to the Sanctuary, used both for cultural and religious purposes and for therapeutic purposes;

- boost the use of the sanctuary by a wider public represented by local, religious and large-scale tourism. 


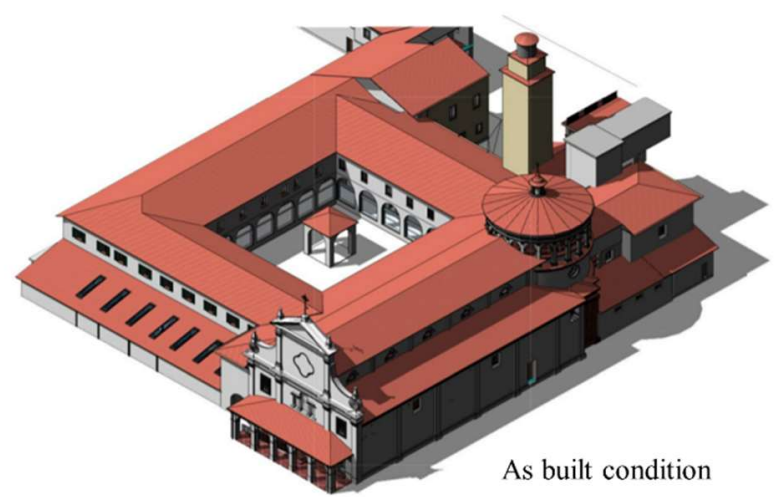

Figure 17. HBIM model in progress, realized on the base of the TLS and UAVs survey.

\section{REFERENCES}

Archivio della Curia Arcivescovile di Milano, 1584. Atti della Visita Apostolica di San Carlo Borromeo al Santuario del Trompone, pp. 1-320.

Besl, P.J. and McKay, N.D., 1992. Method for registration of 3D shapes. In Sensor fusion IV: control paradigms and data structures, Vol. 1611, pp. 586-606. International Society for Optics and Photonics.

Bono Don Pietro, 1935. La Madonna del Trompone, Cigliano, Tipografia legatoria Arturo Stella, pp. 1-155.

Cappellino Don Mario, 1995. Notizie per la storia, la spiritualità, l'arte del Santuario della Madonna del Trompone, ed. Silenzi operai della Croce, Moncrivello (VC), pp. 1-88.

Dissanayake, M. G., Newman, P., Clark, S., Durrant-Whyte, H. F., and Csorba, M., 2001. A solution to the simultaneous localization and map building (SLAM) problem. IEEE Transactions on robotics and automation, 17(3), 229-241.

Chiabrando, F., Della Colletta, C., Sammartano, G., Spanò, A., Spreafico, A., 2018. "Torino 1911" project: a contribution of a slam-based survey to extensive 3D heritage modeling, In: The International Archives of the Photogrammetry, Remote Sensing and Spatial Information Sciences, Volume XLII-2, pp. 225-234. https://doi.org/10.5194/isprs-archives-XLII-2-225-2018

Kaarta, 2018. Kaarta, Instructions for Stencil ${ }^{\circledR}$. Pp. 1-91.

Keller, F.; Sternberg, H., 2013. Multi-sensor platform for indoor mobile mapping: System calibration and using a total station for indoor applications. Remote Sens. 2013, 5, pp. 5805-5824.

Khoshelham, K., Diaz Vilariño, L., Peter, M., Kang, Z., Acharya, D., 2017. The ISPRS Benchmarck on indoor modelling. In: The International Archives of the Photogrammetry, Remote Sensing and Spatial Information Sciences, Volume XLII-2/W7, pp. 367372. https://doi.org/10.5194/isprs-archives-XLII-2-W7-3672017

Lagüela, S.; Dorado, I.; Gesto, M.; Arias, P.; González-Aguilera, D.; Lorenzo, H., 2018. Behavior Analysis of Novel Wearable Indoor Mapping System Based on 3D-SLAM. In: Sensors 18(3):766

https://doi.org/10.3390/s18030766
Lehtola, V., Kaartinen, H., Nüchter, A., Kaijaluoto, R., Kukko, A., Litkey, P., Honkavaara, E., Rosnell, T., Vaaja, M., Virtanen, J.P. and Kurkela, M., 2017. Comparison of the selected state-ofthe-art 3D indoor scanning and point cloud generation methods. Remote sensing, 9(8), p.796.

Nocerino, E., Menna, F., Remondino, F., Toschi, I. and Rodríguez-Gonzálvez, P., 2017. Investigation of indoor and outdoor performance of two portable mobile mapping systems. In Videometrics, Range Imaging, and Applications XIV (Vol. 10332, p. 103320I). International Society for Optics and Photonics.

https://doi.org/10.1117/12.2270761

Nüchter, A., Lingemann, K., Hertzberg, J. and Surmann, H., 2007. 6D SLAM -3D mapping outdoor environments. Journal of Field Robotics, 24(8-9), pp.699-722.

Pauna Don Pietro, 1895. Brevi Cenni storici sul santuario di M. Ss. Del Trompone e dell'unito seminario di Moncrivello, Archidiocesi di Vercelli, Moncrivello, pp. 1-150.

Pissinis M., Carando C., 1988. Architettura religiosa nella diocesi di Vercelli: il Santuario della Madonna del Trompone di Moncrivello. Storia e Riuso, Politecnico di Torino, facoltà di architettura, tesi di Laurea, relatore prof. P. Chierici, pp. 1-97.

Sammartano, G., and Spanò, A., 2018. Point clouds by SLAMbased mobile mapping systems: accuracy and geometric content validation in multisensor survey and stand-alone acquisition. Applied Geomatics, 10(4), 317-339.

Thomson, C.; Apostolopoulos, G.; Backes, D.; Boehm, J., 2013. Mobile laser scanning for indoor modelling. ISPRS Ann. Photogramm. Remote Sens. Spat. Inf. Sci. 2013, II-5/W2, 289293.

Tucci, G., Visintini, D., Bonora, V., and Parisi, E., 2018. Examination of indoor mobile mapping systems in a diversified internal/external test field. Applied Sciences, 8(3), 401.

Zhang, J. and Singh, S., 2018. Laser-visual-inertial odometry and mapping with high robustness and low drift. Journal of Field Robotics, 35(8), pp.1242-1264. 